\title{
NOTE ON THE UNITS OF A REAL QUADRATIC FIELD
}

\author{
S. CHOWLA
}

Let $p$ denote a prime $\equiv 1(\bmod 4)$. It is known that, if $\theta=e^{2 \pi i / p}$,

$$
\frac{\prod_{n}\left(1-\theta^{b}\right)}{\prod_{r}\left(1-\theta^{a}\right)}=\eta
$$

(here $b$ and $a$ run over the quadratic nonresidues and quadratic residues, respectively, that lie between 0 and $p$ ) is a unit of the real quadratic field $R(\sqrt{ } p)$, and that $\eta>1$.

The fact that $\eta>1$ is usually deduced from the theory of the classnumber of quadratic fields. We present a short proof independent of the theory of the class-number. As in the paper of Chowla and Mordell [Note on the nonvanishing of $L(1)$, Proc. Amer. Math. Soc. 12 (1961), 283-284], we have $\eta \neq 1$ since $L(1) \neq 0$ (proved in the paper cited) in the relation [ibid.]

$$
\eta=\exp \{S L(1)\}
$$

where $L(1)=\sum_{1}^{\infty}(n \mid p) n^{-1}$ and $S=\sum_{1}^{p}(n \mid p) \theta^{n}$. Here $(n \mid p)$ is Legendre's symbol.

From the theory of the Gaussian sum,

$$
S=\sqrt{ } p
$$

Also,

$$
L(1)=\lim _{s \rightarrow 1+0} L(s) \geq 0
$$

since

$$
L(s)=\sum_{1}^{\infty}(n \mid p) n^{-s}>0 \quad(s>1)
$$

from the Euler product for $L(s)$. It now follows from Chowla and Mordell that

$$
L(1)>0 .
$$

From (1), (2), (3) we obtain $\eta>1$. Q.E.D.

Pennsylvania State University

Received by the editors January $31,1964$. 